17

\title{
Электронная структура и спектры оптического поглощения золотых фуллеренов $\mathrm{Au}_{16}$ и $\mathrm{Au}_{20}$
}

\author{
(С) Г.И. Миронов \\ Марийский государственный университет, \\ Йошкар-Ола, Россия \\ E-mail: mirgi@marsu.ru \\ Поступила в Редакцию 25 декабря 2018 г. \\ В окончательной редакции 25 декабря 2018 г. \\ Принята к публикации 28 декабря 2018 г.
}

\begin{abstract}
Электронные и оптические свойства золотых фуллеренов изучаются в рамках модели Хаббарда. Получены выражения для фурье-образов антикоммутаторных функций Грина для золотых фуллеренов $\mathrm{Au}_{16}$ и $\mathrm{Au}_{20}$, полюса которых определяют энергетический спектр рассматриваемой системы. Вычислены термодинамические средние, характеризующие перескоки электрона с узла наносистемы на соседний узел, корреляционные функции, свидетельствующие о вероятности нахождения двух $d$-электронов с противоположно ориентированными проекциями спинов на одном узле рассматриваемых фуллеренов из атомов золота. Приведены спектры оптического поглощения. Пики оптического поглощения ионов $\mathrm{Au}_{20}^{-}$и $\mathrm{Au}_{16}^{-}$соответствуют ближней инфракрасной области спектра, где поглощение света кровью или мягкой тканью ничтожно мало, поэтому эти ионы могут привлекаться как новый класс контрастных улучшений и фототерапевтических средств для диагностики и лечения рака.
\end{abstract}

DOI: 10.21883/FTT.2019.06.47700.346

Золотые фуллерены представляют собой полые наноструктуры с пористыми стенками, которые могут быть получены с помощью реакции гальванической замены между серебряными нанокубами и хлорауриновой кислотой [1]. „Первое доказательство существования полых золотых клеток“ $\mathrm{Au}_{16}$ и $\mathrm{Au}_{20}$, названных впоследствии золотыми фуллеренами, было приведено в 2006 году [2]. Исследование наночастиц золота показало, что они обладают уникальными электрофизическими [3-5], оптическими [6,7], электрохимическими [8] свойствами. Еще в конце восьмидесятых годов прошлого столетия в $[9,10]$ было обнаружено, что золотые наночастицы показывают ярко выраженную каталитическую активность при протекании некоторых химических процессов, эти работы вызвали лавинообразный интерес к физике и химии нанокластеров золота, особенно полых наноструктр из атомов золота [6]. Существенно, что оптические свойства золотых фуллеренов делают их весьма привлекательными как для диагностики раковых заболеваний, так и для их лечения [1,11-15].

Вскоре после синтеза золотых фуллеренов [2,6] появились теоретические работы $[16,17]$, в которых были вычислены фурье-образы антикоммутаторных функций Грина для золотого фуллерена $\mathrm{Au}_{16}$, определен энергетический спектр, вычислены энергия ионизации и энергия сродства к электрону. Необычным здесь по аналогии со случаем фуллерена $\mathrm{C}_{60}$ оказалось сочетание высокого, положительного по значению энергии сродства к электрону и относительно низкой энергии ионизации явление редкое в химии и физике, указывающее, что молекулы фуллерена $\mathrm{Au}_{16}$ могут одновременно быть как донорами, так и акцепторами электронов в хи- мических процессах. Полученные результаты позволили в определенной степени понять, почему инертный металл $\mathrm{Au}$ на наноуровне начинает проявлять селективные каталитические свойства (см. [16,17]). Практически одновременно появилась работа [18], в которой численно в рамках теории функционала плотности была определена структура фуллерена $\mathrm{Au}_{20}$ и найдены значения энергии ионизации и энергии сродства к электрону, позволяющие в определенной мере интерпретировать каталитические свойства фуллерена из атомов золота $\mathrm{Au}_{20}$. Результаты, полученные в [16-18], дали возможность объяснить результаты более ранних экспериментальных исследований золотых нанокластеров в [19-21].

Целью настоящей работы является сравнительное теоретическое исследование фуллеренов из атомов золота $\mathrm{Au}_{20}$ и $\mathrm{Au}_{16}$ с учетом того, что эти молекулы являются системами с сильными корреляциями, при этом определяющую роль играют $d$-электроны, поскольку энергетический спектр $d$-электронов оказывается выше спектра $s$-электронов [22,23], в электронном транспорте участвуют только $d$-электроны.

Молекула фуллерена $\mathrm{Au}_{20}$ обладает пирамидальной структурой с тетраэдрической симметрией, модель этого фуллерена приведена, например, в [18], при этом четыре атома золота расположены в центрах четырех треугольных граней, четыре атома занимают четыре угла треугольной пирамиды, остальные 12 атомов находятся на ребрах пирамиды вокруг атома в центре грани. Отличие молекулы фуллерена $\mathrm{Au}_{16}$ (см. рис. 1) заключается в том, что отсутствуют четыре атома, занимающие 4 угла треугольной пирамиды. 


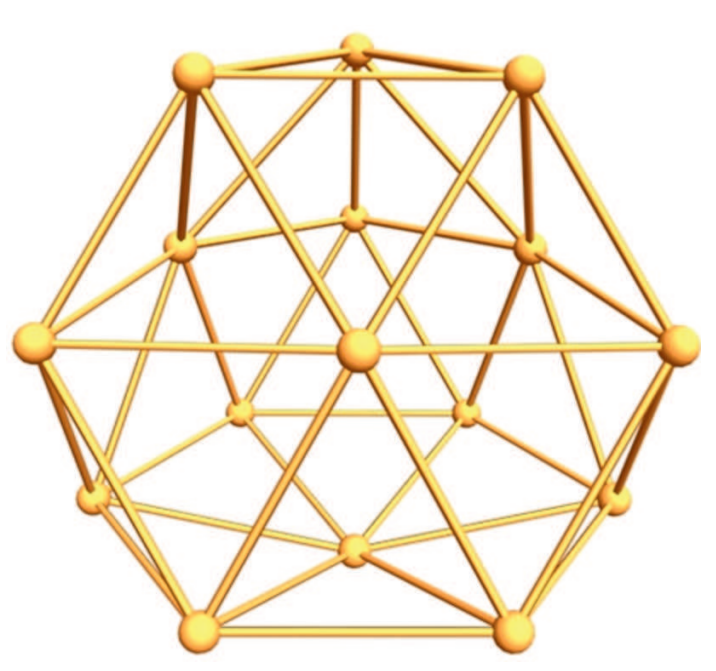

Рис. 1. Модель молекулы фуллерена $\mathrm{Au}_{16}$. В случае модели $\mathrm{Au}_{20}$ добавятся еще 4 атома $\mathrm{Au}$ : один атом к трем атомам сверху в виде вершины пирамиды, по одному атому к треугольникам снизу слева и снизу справа, а также к трем атомам на заднем плане в виде угловых атомов, образующих основание пирамиды.

Для изучения систем, в которых определяющую роль играют $d$-электроны, более полувека назад была предложена модель Хаббарда [24]. Отметим, что за 30 лет до работы Хаббарда предлагалась более общая модель [25] Шубина-Вонсовского, модель Хаббарда является частным случаем модели, предложенной в [24]. В рамках гамильтониана Хаббарда атом металла заменяется моделью, в которой вокруг положительно заряженного иона движется электрон. В зависимости от степени локализации или делокализации этого электрона как основной результат получается фазовый переход металл-диэлектрик, удается объяснить наиболее существенные свойства переходных металлов, а также понять причины особенностей свойств наноструктур $[16,17]$.

Гамильтониан модели Хаббарда рассматриваемых наносистем имеет вид:

$$
\begin{gathered}
\hat{H}=\hat{H}_{0}+\hat{V}, \\
\hat{H}_{0}=\sum_{\sigma, f=1}^{N} \varepsilon n_{f \sigma}+\sum_{\sigma, f \neq l} B_{f l}\left(a_{f \sigma}^{+} a_{l \sigma}+a_{l \sigma}^{+} a_{f \sigma}\right), \\
\hat{V}=U \sum_{f=1}^{N} \hat{n}_{f \uparrow} \hat{n}_{f \downarrow},
\end{gathered}
$$

где $a_{j \sigma}^{+}, a_{j \sigma}-$ ферми-операторы рождения и уничтожения электронов на узле $j(j=f, l)$ золотого фуллерена с проекцией спина $\sigma(\sigma=\uparrow, \downarrow) . \hat{n}_{j \sigma}=a_{j \sigma}^{+} a_{j \sigma}$ - оператор числа частиц, $\varepsilon-$ собственная энергия электрона, $U$ - энергия кулоновского отталкивания электронов с противоположно ориентированными проекциями спинов на одной орбитали, $B_{f l}=B(f-l)-$ интеграл переноса. $N$ в (2)-(3) принимает значение 16 в случае $\mathrm{Au}_{16}$ и 20 в случае молекулы $\mathrm{Au}_{20}$.

Методика решения модели Хаббарда, вычисления фурье-образа антикоммутаторной функции Грина применительно к наносистемам подробно приведена в работах $[16,17]$, поэтому сразу приведем формулу для фурьеобраза антикоммутаторной функции Грина фуллерена $\mathrm{Au}_{20}$ для атома в центре правильного шестиугольника, составленного из треугольников, атом находится на треугольной грани пирамиды:

$$
\begin{aligned}
& \left\langle\left\langle a_{1 \uparrow}^{+} \mid a_{1 \uparrow}\right\rangle\right\rangle_{E}=\frac{i}{2 \pi} \sum_{n=1}^{2}\left\{\frac{0.0235}{E-\varepsilon_{n}+1.3615 B}\right. \\
& +\frac{0.1405}{E-\varepsilon_{n}-0.8326 B}+\frac{0.0610}{E-\varepsilon_{n}-3.5289 B}+\frac{0.0250}{E-\varepsilon_{n}} \\
& \left.+\frac{0.1500}{E-\varepsilon_{n}+2 B}+\frac{0.0319}{E-\varepsilon_{n}-5.6533 B}+\frac{0.0681}{E-\varepsilon_{n}+2.6533 B}\right\} .
\end{aligned}
$$

В случае аналогичного атома в $\mathrm{Au}_{16}$ будем иметь

$$
\begin{aligned}
\left\langle\left\langle a_{1 \uparrow}^{+} \mid a_{1 \uparrow}\right\rangle\right\rangle_{E}= & \frac{i}{2 \pi} \sum_{n=1}^{2}\left\{\frac{0.0377}{E-\varepsilon_{n}-5.2749 B}\right. \\
& +\frac{0.0873}{E-\varepsilon_{n}+2.2749 B}+\frac{0.1000}{E-\varepsilon_{n}-3 B} \\
& \left.+\frac{0.1250}{E-\varepsilon_{n}}+\frac{0.1500}{E-\varepsilon_{n}+2 B}\right\} .
\end{aligned}
$$

В формулах (4) и (5) $\varepsilon_{1}=\varepsilon+U, \varepsilon_{2}=\varepsilon$. Отметим, что в формулах (4)-(5) полюса фурье-образов функций Грина определяют энергетический спектр фуллерена $\mathrm{Au}_{20}$ и $\mathrm{Au}_{16}$ соответственно числители - вероятности нахождения электронов на соответствующих уровнях энергии. Из анализа формул (4) и (5) следует, что четыре уровня энергии в спектрах $\mathrm{Au}_{20}$ и $\mathrm{Au}_{16}$ являются одинаковыми, это уровни энергии $\varepsilon, \varepsilon+U, \varepsilon-2 B, \varepsilon+U-2 B$, причем вероятность обнаружения электрона на уровнях энергии $\varepsilon$ и $\varepsilon+U$ в случае фуллерена $\mathrm{Au}_{16}$ в пять раз больше по сравнению с фуллереном $\mathrm{Au}_{20}$. Уровни энергии $\varepsilon-2 B, \varepsilon+U-2 B$ оказываются занятыми электронами с одинаковыми вероятностями 0.15 .

На рис. 2 приведены энергетические спектры в случае рассматриваемых атомов, описываемых индексом 1 , для значений параметров моделей золотых фуллеренов $U=8.85 \mathrm{eV}, B=-1 \mathrm{eV}, \varepsilon=-2.95 \mathrm{eV}$.

Энергетические спектры состоят из двух подзон верхней хаббардовской подзоны (некий аналог зоны проводимости) и нижней хаббардовской подзоны (аналог валентной зоны). В основном состоянии, когда в среднем на каждый узел наносистемы приходится один электрон, нижняя подзона заполнена полностью, в верхней подзоне в основном состоянии электроны отсутствуют. $\mathrm{B}$ случае $\mathrm{Au}_{16}$ пять нижних уровней энергии образуют 


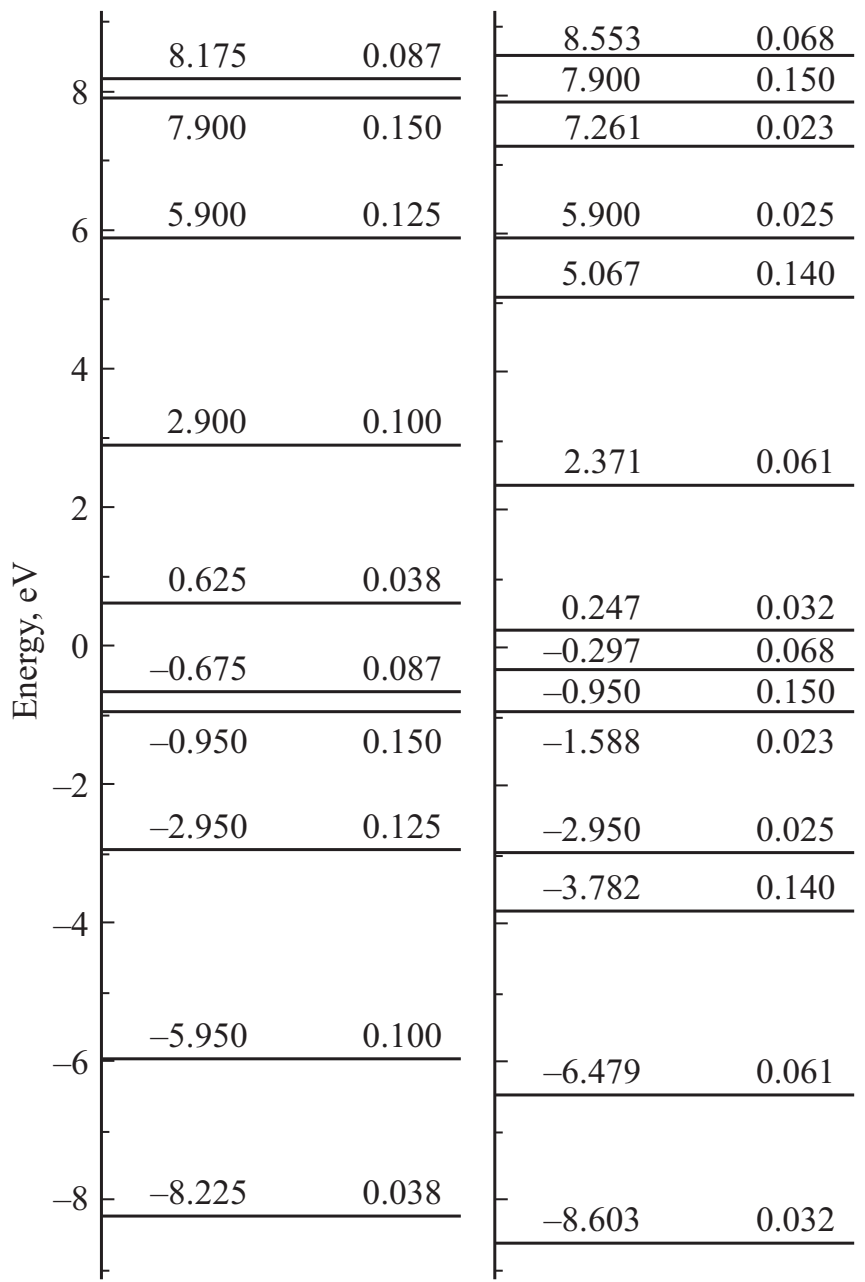

Рис. 2. Энергетический спектр фуллеренов $\mathrm{Au}_{16}$ (слева) и $\mathrm{Au}_{20}$ (справа) при значениях параметров системы: $U=8.85 \mathrm{eV}, B=-1 \mathrm{eV}, \varepsilon=-2.95 \mathrm{eV}$.

нижнюю хаббардовскую подзону, пять верхних - верхнюю хаббардовскую подзону. Ширина каждой подзоны равна $7.55 \mathrm{eV}$, ширина зоны запрещенных энергий равна $1.3 \mathrm{eV}$. В случае $\mathrm{Au}_{20}$ нижняя хаббардовская подзона состоит из семи нижних уровней энергии, верхняя из семи верхних уровней энергии. Ширины подзон одинаковы и равны $8.31 \mathrm{eV}$, ширина запрещенной зоны равна $0.54 \mathrm{eV}$. Таким образом, при рассматриваемых значениях параметров анализируемые наносистемы ведут себя как полупроводники. На рис. 2 над уровнями энергии (в некоторых случаях под уровнями энергии) приведены значения энергий, соответствующие этим уровням, и вероятности нахождения электронов на этих уровнях энергии. Например, в случае $\mathrm{Au}_{16}$ электрон занимает на первом узле наносистемы уровень энергии $8.175 \mathrm{eV}$ с вероятностью 0.087. Понятно, что если просуммировать вероятности нахождения электронов на всех уровнях энергии, мы получим вероятность достоверного события.
Возьмем теперь в $\mathrm{Au}_{20}$ произвольный атом рядом с атомом 1, который будет находиться на ребре пирамиды. Обозначим этот атом как второй атом, фурье-образ функции Грина будет иметь вид

$$
\begin{aligned}
& \left\langle\left\langle a_{2 \uparrow}^{+} \mid a_{2 \uparrow}\right\rangle\right\rangle_{E}=\frac{i}{2 \pi} \sum_{n=1}^{2}\left\{\frac{0.0480}{E-\varepsilon_{n}+1.3615 B}\right. \\
& +\frac{0.0400}{E-\varepsilon_{n}-0.8326 B}+\frac{0.0870}{E-\varepsilon_{n}-3.5289 B}+\frac{0.0833}{E-\varepsilon_{n}} \\
& \left.+\frac{0.2000}{E-\varepsilon_{n}+2 B}+\frac{0.0284}{E-\varepsilon_{n}-5.6533 B}+\frac{0.0133}{E-\varepsilon_{n}+2.6533 B}\right\} .
\end{aligned}
$$

Для аналогичного атома в фуллерене $\mathrm{Au}_{16}$ получим:

$$
\begin{aligned}
\left\langle\left\langle a_{2 \uparrow}^{+} \mid a_{2 \uparrow}\right\rangle\right\rangle_{E}= & \frac{i}{2 \pi} \sum_{n=1}^{2}\left\{\frac{0.0291}{E-\varepsilon_{n}-5.2749 B}\right. \\
& +\frac{0.0125}{E-\varepsilon_{n}+2.2749 B}+\frac{0.0917}{E-\varepsilon_{n}-3 B} \\
& \left.+\frac{0.1667}{E-\varepsilon_{n}}+\frac{0.2000}{E-\varepsilon_{n}+2 B}\right\} .
\end{aligned}
$$

Сравнение формул (6) и (4), показывает интересную особенность наносистем: хотя энергетический спектр для электронов, находящихся на узлах 1 и 2 одинаков, вероятности нахождения электронов на этих уровнях энергий отличаются. Например, электрон на узле 1 молекулы фуллерена $\mathrm{Au}_{20}$ может находиться на уровне энергии $E=\varepsilon-2 B$ с вероятностью 0.15 , тогда как на соседнем атоме 2 этого же фуллерена электрон может быть обнаружен с вероятностью 0.2 на этом же уровне энергии $E=\varepsilon-2 B$. То же самое можно обнаружить при сравнении формул (5) и (7) в случае $\mathrm{Au}_{16}$.

Для атома, занимающего угол пирамиды в фуллерене $\mathrm{Au}_{20}$, получим следующую формулу для фурье-образа функции Грина

$$
\begin{aligned}
& \left\langle\left\langle a_{3 \uparrow}^{+} \mid a_{3 \uparrow}\right\rangle\right\rangle_{E}=\frac{i}{2 \pi} \sum_{n=1}^{2}\left\{\frac{0.2075}{E-\varepsilon_{n}+1.3615 B}\right. \\
& +\frac{0.1144}{E-\varepsilon_{n}-0.8326 B}+\frac{0.0530}{E-\varepsilon_{n}-3.5289 B}+\frac{0.1000}{E-\varepsilon_{n}} \\
& \left.+\frac{0.0000}{E-\varepsilon_{n}+2 B}+\frac{0.0080}{E-\varepsilon_{n}-5.6533 B}+\frac{0.0170}{E-\varepsilon_{n}+2.6533 B}\right\} .
\end{aligned}
$$

Если взять тот же самый уровень энергии $E=\varepsilon-2 B$, электрон, занимая любой из четырех угловых атомов пирамиды, не может находиться на этом уровне энергии, с конечными вероятностями мы можем обнаружить электроны лишь на оставшихся 12 уровнях энергии. $\mathrm{B} \mathrm{Au}_{16}$ атом подобного сорта отсутствует. 
Величина собственной энергии $\varepsilon$ зависит от параметров системы $U, B$ и от числа электронов в системе $N_{e}$. Для определения этой величины необходимо написать и решить уравнение на химический потенциал. Сделаем это следующим образом. Используя флуктуационнодиссипационную теорему [26] из фурье-образа функции Грина (4) можем получить следующее термодинамическое среднее, описывающее среднее количество электронов на узле 1 с проекцией спина вверх

$$
\begin{aligned}
& \left\langle\hat{n}_{1 \uparrow}\right\rangle=\sum_{n=1}^{2}\left\{0.0235 f^{+}\left(\varepsilon_{n}-1.3615 B\right)\right. \\
& +0.1405 f^{+}\left(\varepsilon_{n}+0.8326 B\right)+0.061 f^{+}\left(\varepsilon_{n}+3.5289 B\right) \\
& +0.025 f^{+}\left(\varepsilon_{n}\right)+0.15 f^{+}\left(\varepsilon_{n}-2 B\right) \\
& \left.+0.0139 f^{+}\left(\varepsilon_{n}+5.6533 B\right)+0.0681 f^{+}\left(\varepsilon_{n}-2.6533 B\right)\right\} .
\end{aligned}
$$

Здесь $f^{+}(x)=(1+\exp (\beta \cdot x))^{-1}, \beta=1 / k T$ - обратная температура. Аналогично для атомов типа 2 и 3 :

$$
\begin{aligned}
& \left\langle\hat{n}_{2 \uparrow}\right\rangle=\sum_{n=1}^{2}\left\{0.048 f^{+}\left(\varepsilon_{n}-1.3615 B\right)\right. \\
& +0.04 f^{+}\left(\varepsilon_{n}+0.8326 B\right)+0.087 f^{+}\left(\varepsilon_{n}+3.5289 B\right) \\
& +0.0833 f^{+}\left(\varepsilon_{n}\right)+0.2 f^{+}\left(\varepsilon_{n}-2 B\right) \\
& \left.+0.0284 f^{+}\left(\varepsilon_{n}+5.6533 B\right)+0.0133 f^{+}\left(\varepsilon_{n}-2.6533 B\right)\right\} . \\
& \left\langle\hat{n}_{3 \uparrow}\right\rangle=\sum_{n=1}^{2}\left\{0.2075 f^{+}\left(\varepsilon_{n}-1.3615 B\right)\right. \\
& +0.1144 f^{+}\left(\varepsilon_{n}+0.8326 B\right)+0.053 f^{+}\left(\varepsilon_{n}+3.5289 B\right) \\
& +0.1 f^{+}\left(\varepsilon_{n}\right)+0.008 f^{+}\left(\varepsilon_{n}+5.6533 B\right) \\
& \left.+0.017 f^{+}\left(\varepsilon_{n}-2.6533 B\right)\right\} .
\end{aligned}
$$

В случае отсутствия внешних или локальных магнитных полей среднее количество электронов на одном узле с проекциями спина „вверх“ и „вниз“ будет одинаковым, поэтому общее количество электронов $N_{e}$ в фуллерене $\mathrm{Au}_{20}$

$$
N_{e}=4 \cdot 2\left\langle\hat{n}_{1 \uparrow}\right\rangle+12 \cdot 2\left\langle\hat{n}_{2 \uparrow}\right\rangle+4 \cdot 2\left\langle\hat{n}_{3 \uparrow}\right\rangle .
$$

Подставляя формулы (8), (9), (10) в выражение (11) мы получим уравнение на химический потенциал, графическое решение которого в случае фуллерена $\mathrm{Au}_{20}$ изображено на рис. 3. Из анализа рисунка следует,

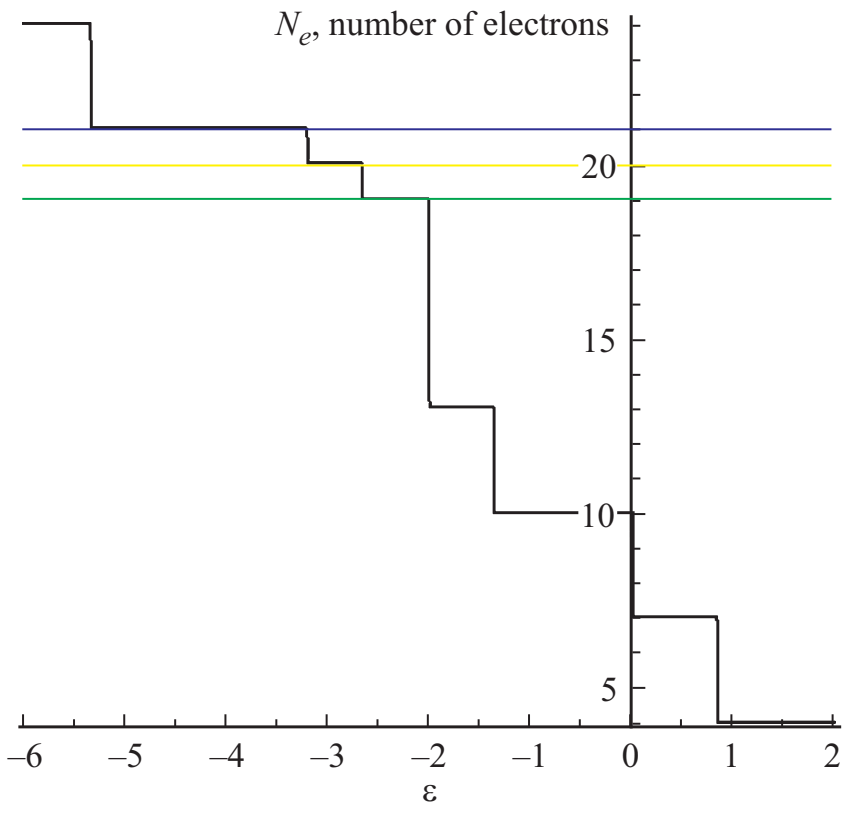

Рис. 3. Решение уравнения на химпотенциал. Зависимость общего числа электронов в фуллерене из 20 атомов золота от собственной энергии при значениях параметров системы: $U=8.85 \mathrm{eV}, B=-1 \mathrm{eV}$.

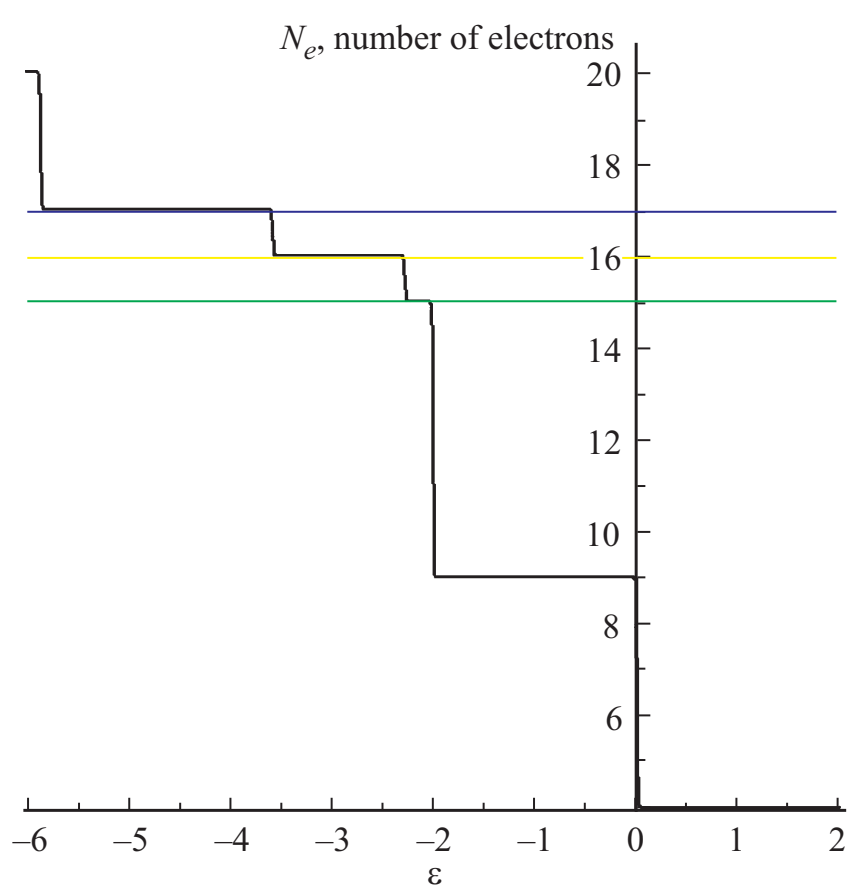

Рис. 4. Решение уравнения на химпотенциал. Зависимость общего числа электронов в фуллерене из 16 атомов золота от собственной энергии при значениях параметров системы: $U=8.85 \mathrm{eV}, B=-1 \mathrm{eV}$

что случаю, когда на 20 узлов фуллерена приходятся 20 электронов, соответствует величина $\varepsilon=-2.95 \mathrm{eV}$, а не $\varepsilon=-U / 2$. На графике можно взять любое значение для $\varepsilon$ в пределах „ступеньки“ для числа электронов 20. 

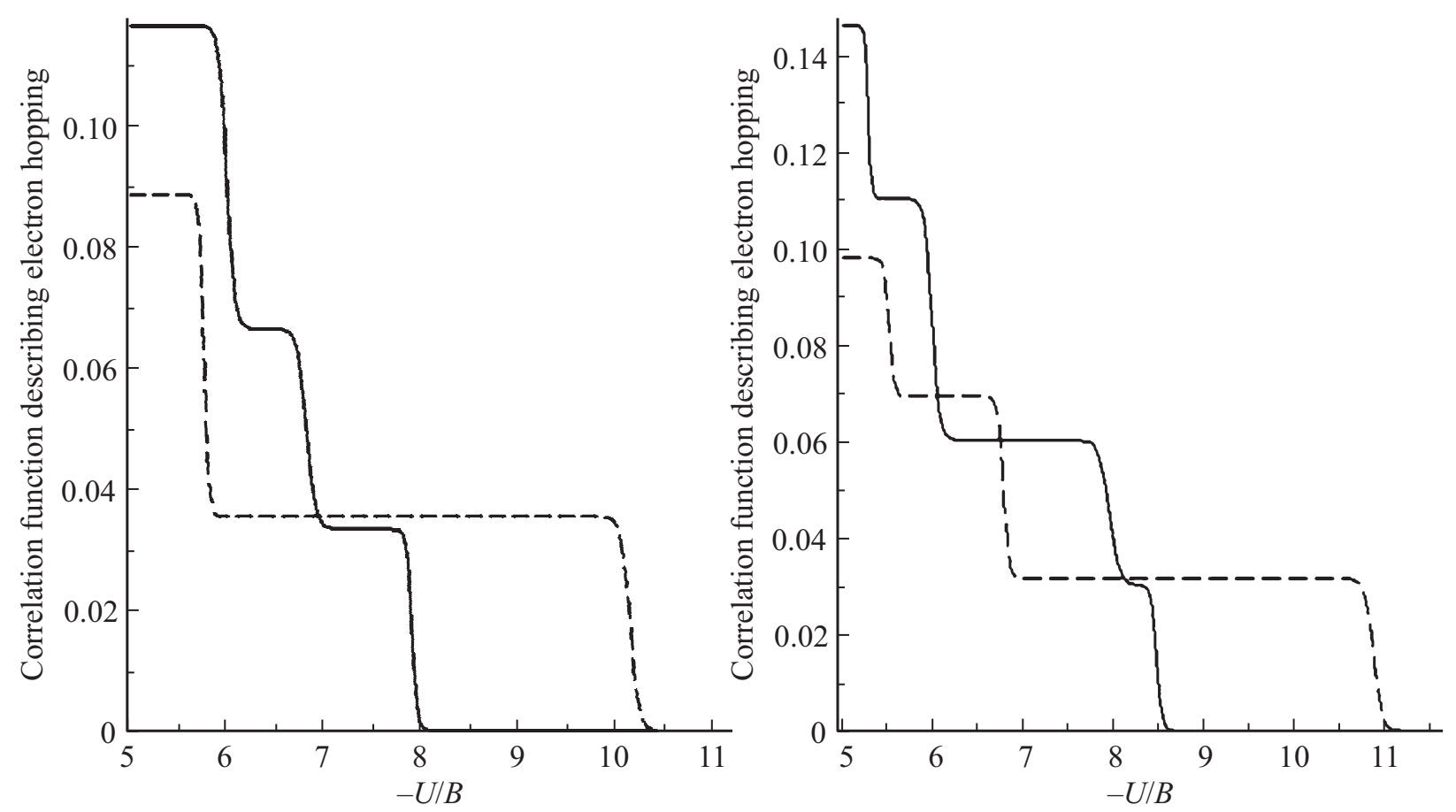

Рис. 5. Зависимость корреляционной функции $\left\langle a_{1 \uparrow}^{+} a_{2 \uparrow}\right\rangle$ от отношения кулоновского потенциала к интегралу переноса, описывающей перескоки электронов со второго узла золотого фуллерена на первый узел. Слева - в случае Аи 16 сплошная линия описывает электронейтральный фуллерен, пунктирная - ион $\mathrm{Au}_{16}^{-}$, содержащий 17 электронов. Справа — в случае Аu 20 сплошная линия описывает электронейтральный фуллерен, пунктирная — ион $\mathrm{Au}_{20}^{-}$, содержащий 21 электрон.

В случае 21 электрона, когда фуллерен $\mathrm{Au}_{20}$ выступает в качестве акцептора, для $\mathrm{Au}_{20}^{-}$мы получим $\varepsilon=-4.25 \mathrm{eV}$. Эти значения собственных энергий нам нужны для расчета термодинамических средних, а также энергий основного состояния.

На рис. 4 приведен вид уравнения химпотенциала для фуллерена $\mathrm{Au}_{16}$. В случае нейтрального фуллерена $\mathrm{Au}_{16} \varepsilon=-2.95 \mathrm{eV}$, а для иона $\mathrm{Au}_{16}^{-}$мы будем иметь $\varepsilon=-4.75 \mathrm{eV}$.

Имея решения для операторов рождения частиц, можно вычислить корреляционные функции, описывающие перескоки электрона с одного узла на другой узел, например перескок электрона со второго узла на первый в случае молекулы $\mathrm{Au}_{16}$ :

$$
\begin{aligned}
\left\langle a_{1 \uparrow}^{+} a_{2 \uparrow}\right\rangle & =\sum_{n=1}^{2}\left\{0 . 0 3 3 1 \left(f^{+}\left(\varepsilon_{n}+5.2749 B\right)\right.\right. \\
& \left.-f^{+}\left(\varepsilon_{n}-2.2749 B\right)\right) \\
& \left.+0.05\left(\left(f^{+}\left(\varepsilon_{n}+3 B\right)-f^{+}\left(\varepsilon_{n}-2 B\right)\right)\right)\right\} .
\end{aligned}
$$

Формулу, подобную (12), можно написать и для других перескоков электрона с узла наносистемы $\mathrm{Au}_{16}$ на соседний узел.

Аналогичным образом можно написать корреляционную функцию, описывающую переход электрона со второго узла наносистемы на первый, и в случае фуллерена $\mathrm{Au}_{20}$

$$
\begin{aligned}
& \left\langle a_{1 \uparrow}^{+} a_{2 \uparrow}\right\rangle=\sum_{n=1}^{2}\left\{0 . 0 3 \left[f^{+}\left(\varepsilon_{n}+5.6553 B\right)\right.\right. \\
& \left.-f^{+}\left(\varepsilon_{n}-2.6553 B\right)\right]-0.0053 f^{+}\left(\varepsilon_{n}-1.3615 B\right) \\
& +0.0195 f^{+}\left(\varepsilon_{n}+0.8326 B\right) \\
& \left.+0.0358 f^{+}\left(\varepsilon_{n}+3.5289 B\right)-0.05 f^{+}\left(\varepsilon_{n}-2 B\right)\right\} .
\end{aligned}
$$

Формулу типа (13) можем вывести и для других типов перескоков электрона в фуллерене $\mathrm{Au}_{20}$.

На рис. 5 приведена зависимость корреляционной функции $\left\langle a_{1 \uparrow}^{+} a_{2 \uparrow}\right\rangle$ от отношения кулоновского потенциала к интегралу переноса, описывающей перескоки электронов со второго узла золотого фуллерена на первый узел. Слева на рисунке в случае $\mathrm{Au}_{16}$ сплошная линия описывает электронейтральный фуллерен, пунктирная - ион $\mathrm{Au}_{16}^{-}$, содержащий 17 электронов. Справа в случае $\mathrm{Au}_{20}$ сплошная линия описывает электронейтральный фуллерен, пунктирная - ион $\mathrm{Au}_{20}^{-}$, содержащий 21 электрон. Из анализа рис. 5 следует, что:

1. Наличие большего числа узлов в случае фуллерена из 20 атомов золота по сравнению с фуллереном из 16 атомов позволяет электронам перескакивать от узла 

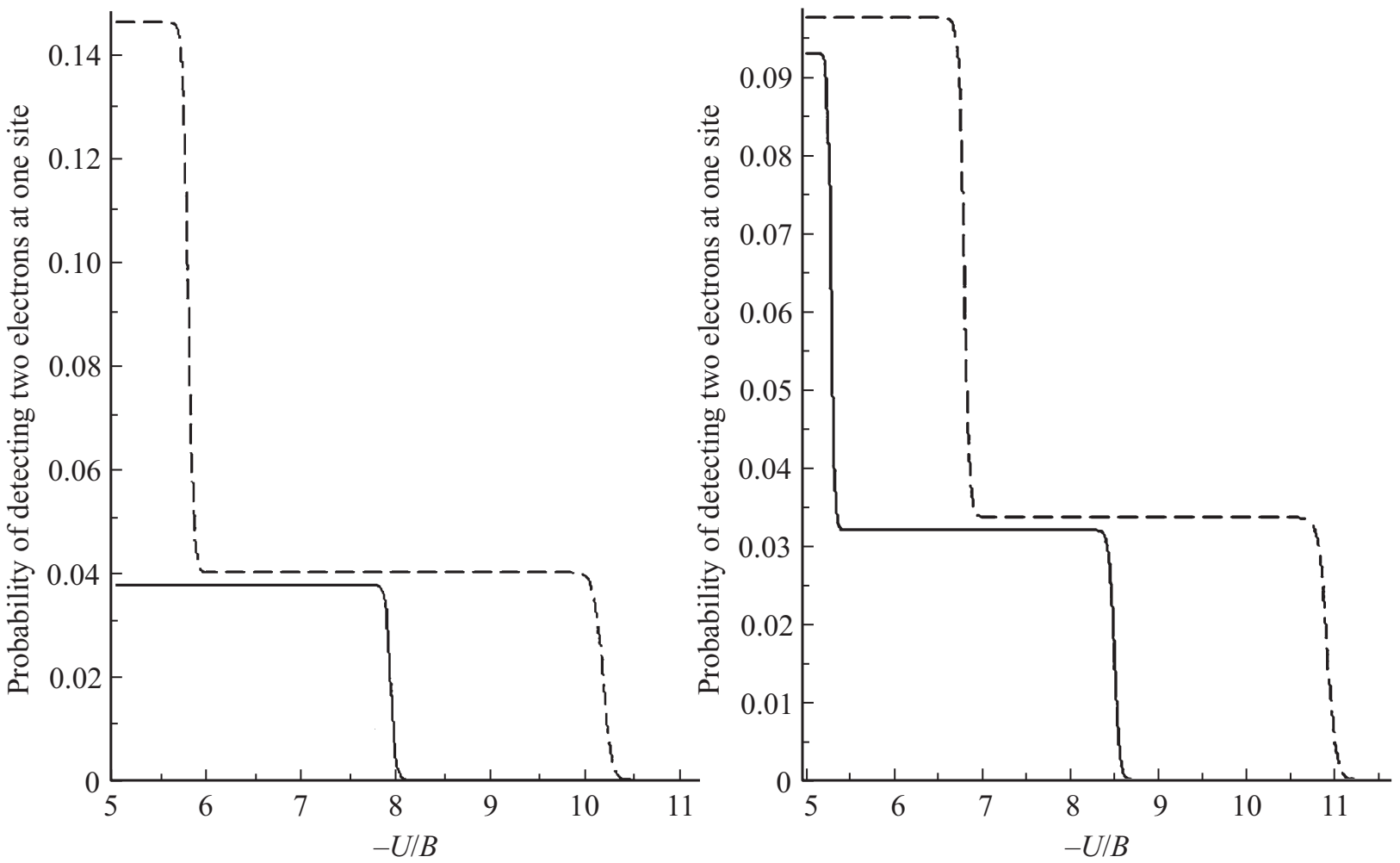

Рис. 6. Зависимость корреляционной функции $\left\langle\hat{n}_{1 \uparrow} n_{1 \downarrow}\right\rangle-$ от отношения кулоновского потенциала к интегралу переноса, описывающей обнаружения двух электронов на одном узле фуллерена. Слева - в случае Аu 16 сплошная линия описывает электронейтральный фуллерен, пунктирная - ион $\mathrm{Au}_{16}^{-}$, содержащий 17 электронов. Справа - в случае $\mathrm{Au}_{20}$ сплошная линия описывает электронейтральный фуллерен, пунктирная — ион $\mathrm{Au}_{20}^{-}$, содержащий 21 электрон.

к узлу при больших значениях кулоновского потенциала при фиксированном значении интеграла переноса. Например, если в $\mathrm{Au}_{16}$ переносы электронов становятся невозможными при $U \approx 8 \mathrm{eV}$, то в $\mathrm{Au}_{20}$ перескоки электронов осуществимы даже при $U \approx 8.5 \mathrm{eV}$.

2. В случае электроотрицательного иона фуллерена наличие одного „лишнего“ электрона приводит к увеличению вероятности перескока с узла на узел даже в области $U \approx 10 \mathrm{eV}$, поскольку появление дополнительного электрона увеличивает интенсивность туннелирования электронов от узла к соседнему узлу.

Таким образом, появление дополнительного электрона в электроотрицательных ионах фуллерена заставляет электроны более интенсивно перемещаться от одного узла к другому. Рассмотрим теперь как в случае ионов $\mathrm{Au}_{16}^{-}, \mathrm{Au}_{20}^{-}$изменится вероятность обнаружения на одном узле, например, первом двух $d$-электронов с противоположно ориентированными проекциями спинов.

Для этого необходимо вычислить корреляционные функции типа $\left\langle\hat{n}_{1 \uparrow} n_{1 \downarrow}\right\rangle$. В случае фуллерена $\mathrm{Au}_{16}$ получим

$$
\begin{aligned}
& \left\langle\hat{n}_{1 \uparrow} n_{1 \downarrow}\right\rangle=0.2243 f^{+}(\varepsilon+U)+0.15 f^{+}(\varepsilon+U-2 B) \\
& +0.1 f^{+}(\varepsilon+U+3 B)+0.0377 f^{+}(\varepsilon+U+5.2749 B) \\
& +0.0873 f^{+}(\varepsilon+U-2.2749 B) .
\end{aligned}
$$

Для других узлов можем получить аналогичные (14) выражения. Если же взять фуллерен $\mathrm{Au}_{20}$, корреляционная функция $\left\langle\hat{n}_{1 \uparrow} n_{1 \downarrow}\right\rangle$ будет иметь вид:

$$
\begin{aligned}
& \left\langle\hat{n}_{1 \uparrow} n_{1 \downarrow}\right\rangle=0.0235 f^{+}(\varepsilon+U-1.3614 B) \\
& +0.1405 f^{+}(\varepsilon+U+0.8326 B) \\
& +0.0609 f^{+}(\varepsilon+U+3.5289 B)+0.025 f^{+}(\varepsilon+U) \\
& +0.15 f^{+}(\varepsilon+U-2 B)+0.0319 f^{+}(\varepsilon+U+5.6533 B) \\
& +0.0681 f^{+}(\varepsilon+U-2.6533 B) .
\end{aligned}
$$

Графики зависимостей $\left\langle\hat{n}_{1 \downarrow} n_{1 \uparrow}\right\rangle$ от величин $U$ и $B$ в областях промежуточной и сильной связей представлены на рис. 6 - слева в случае $\mathrm{Au}_{16}$, справа для $\mathrm{Au}_{20}$. В области сильных корреляций дополнительный электрон в ионах $\mathrm{Au}_{16}^{-}, \mathrm{Au}_{20}^{-}$приводит как к увеличению вероятности обнаружения двух электронов на узле 1, так и к смещению величины кулоновского потенциала $U$ вправо при фиксированной величине интеграла переноса $B$. Понятно, что имеется определенная корреляция в поведениях термодинамических средних, описывающих переносы электронов и вероятности обнаружения двух электронов на узле наносистем. Рассмотрим далее, как поведение рассмотренных корреляционных функций скажется на энергии основного состояния золотых 

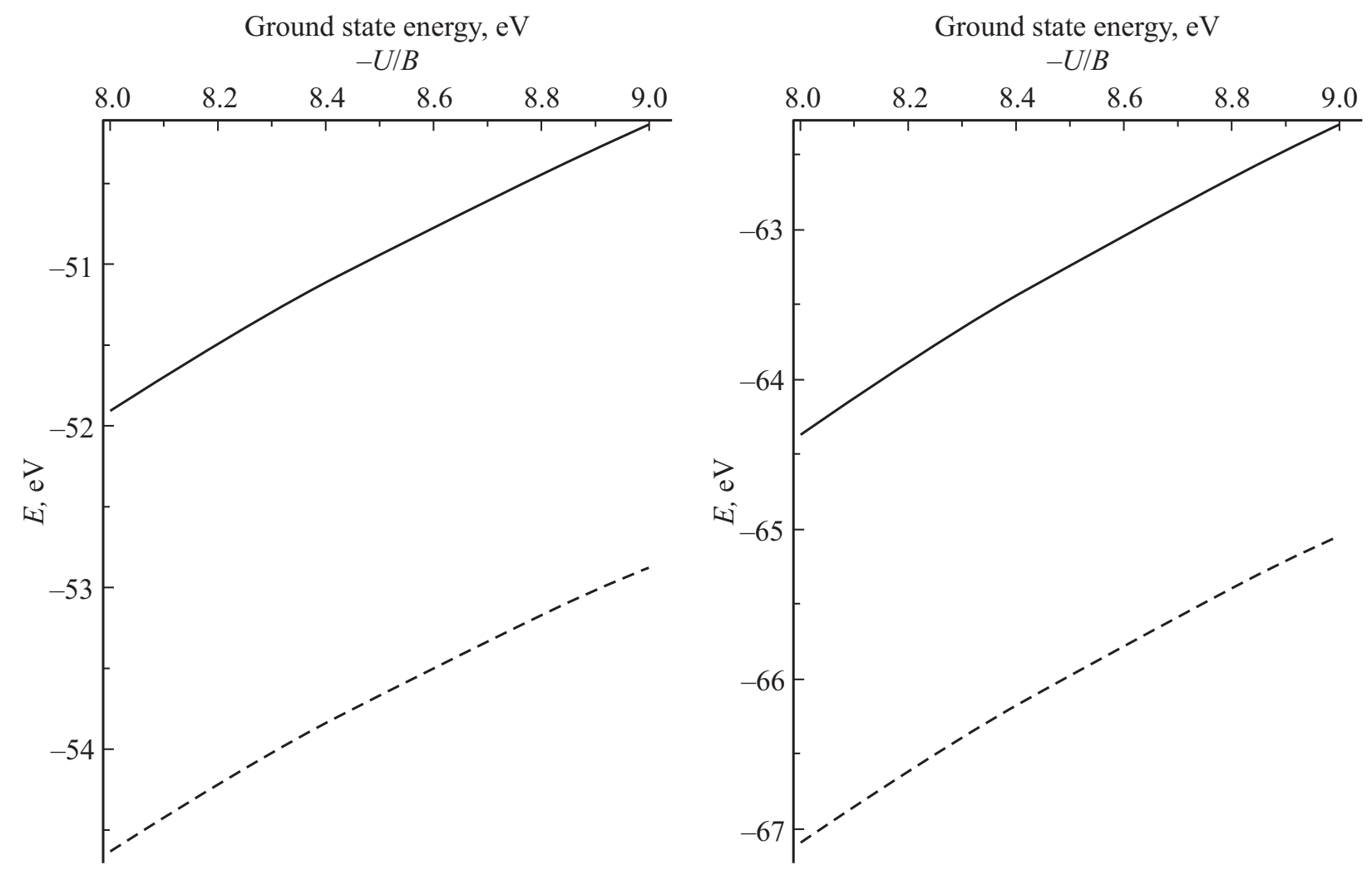

Рис. 7. Зависимость среднего значения энергии фуллеренов от отношения $-U / B$. Слева - в случае фуллерена $А u_{16}-$ сплошная

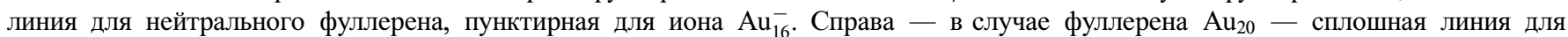
нейтрального фуллерена, пунктирная для иона $\mathrm{Au}_{20}^{-}$.

фуллеренов и, как следствие, на их физико-химических свойствах.

Осуществив термодинамическое усреднение оператора Гамильтона (1)-(3) мы получим выражение для расчета среднего значения энергии наносистемы, при температуре $T \rightarrow 0$ эту энергию называют энергией основного состояния. На рис. 7 слева изображен график зависимости среднего значения энергии фуллерена из 16 атомов золота. Сплошной график соответствует нейтральной молекуле $\mathrm{Au}_{16}$, пунктирный — анионному нанокластеру $\mathrm{Au}_{16}^{-}$. Справа показана зависимость энергии фуллерена из 20 атомов золота. Сплошной график соответствует нейтральной молекуле $\mathrm{Au}_{20}$, пунктирный электроотрицательной наносистеме $\mathrm{Au}_{20}^{-}$. Из анализа приведенных графиков можно сделать вывод о том, что анионное состояние фуллеренов более выгодно, оно является более стабильным по сравнению с электрически нейтральным состоянием, поскольку энергия отрицательно заряженных систем $\mathrm{Au}_{16}^{-}$и $\mathrm{Au}_{20}^{-}$лежит ниже энергии электронейтральных наносистем. Это объясняется тем, что ,дополнительные“ семнадцатый в $\mathrm{Au}_{16}$ и двадцать первый в $\mathrm{Au}_{20}$ электроны вызывают необходимость более интенсивно переходить от атома к атому. Более интенсивные перескоки электронов от узла к узлу и понижают энергию основного состояния фуллеренов, увеличивают значение энергии связи в рассматриваемых наносистемах. Энергия связи электронейтральной $\mathrm{Au}_{20}$ является достаточно исследованной величиной. В [27] получено, что энергия связи молекулы $\mathrm{Au}_{20}$ равна $63.46 \mathrm{eV}$. Согласно работе [28] энергия связи равна $51.63 \mathrm{eV}$. Согласно нашей работе из сплошной кривой справа при значениях параметров $U=8.85 \mathrm{eV}, B=-14 \mathrm{eV}$ следует, что энергия связи равна $62.56 \mathrm{eV}$. Из анализа пунктирного графика при тех же значениях параметров мы можем сделать прогноз, что энергия связи аниона $\mathrm{Au}_{20}^{-}$равна $65.31 \mathrm{eV}$. Из этих двух значений можно вычислить энергию сродства к электрону: $E_{A}=2.75 \mathrm{eV}$. Энергия сродства к электрону это энергия, которая выделится при присоединении к нейтральному фуллерену $\mathrm{Au}_{20}$ дополнительного электрона. Экспериментально энергию связи можно определить, произведя отрыв от аниона $\mathrm{Au}_{20}^{-}$электрона с помощью лазерного излучения. Известны следующие экспериментальные значения энергии сродства к электрону в случае фуллерена из 20 атомов золота:

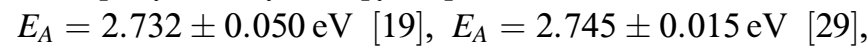
$E_{A}=2.751 \pm 0.010 \mathrm{eV}[1]$. Таким образом, наши результаты для энергии связи и энергии сродства к электрону соответствуют известным результатам. Отметим, что в случае фуллерена $\mathrm{Au}_{16}$ из анализа графиков на левой стороне на рис. 7 энергия связи получается 
равной $50.36 \mathrm{eV}$, энергия сродства к электрону — равной $2.74 \mathrm{eV}$.

Рассмотрим теперь оптические свойства рассматриваемых фуллеренов. Для этого с учетом того, что в $\mathrm{Au}_{16}$ имеются два типа узлов (один тип узлов имеет координационное число 6 , другой тип - пять), энергетический спектр представим в виде, показанном на рис. 8. На рис. 8 слева мы имеем спектр элементарных возбуждений $\mathrm{Au}_{16}$, около уровней энергий наряду со значениями энергий приведены кратность вырождения и значение орбитального квантового числа $l$. Например, уровень энергии $E=-2.95 \mathrm{eV}$ характеризуется кратностью вырождения 5 и орбитальным квантовым числом $l=2$. В случае сильно коррелированных систем это означает, что на этом уровне энергии могут находиться 5 электронов с произвольными $(\uparrow, \downarrow)$ проекциями спинов на ось $O Z$. Если просуммировать все кратности вырождения для уровней энергий, принадлежащих нижней хаббардовской подзоне, то получим, что 16 электронов в основном состоянии занимают все уровни валентной

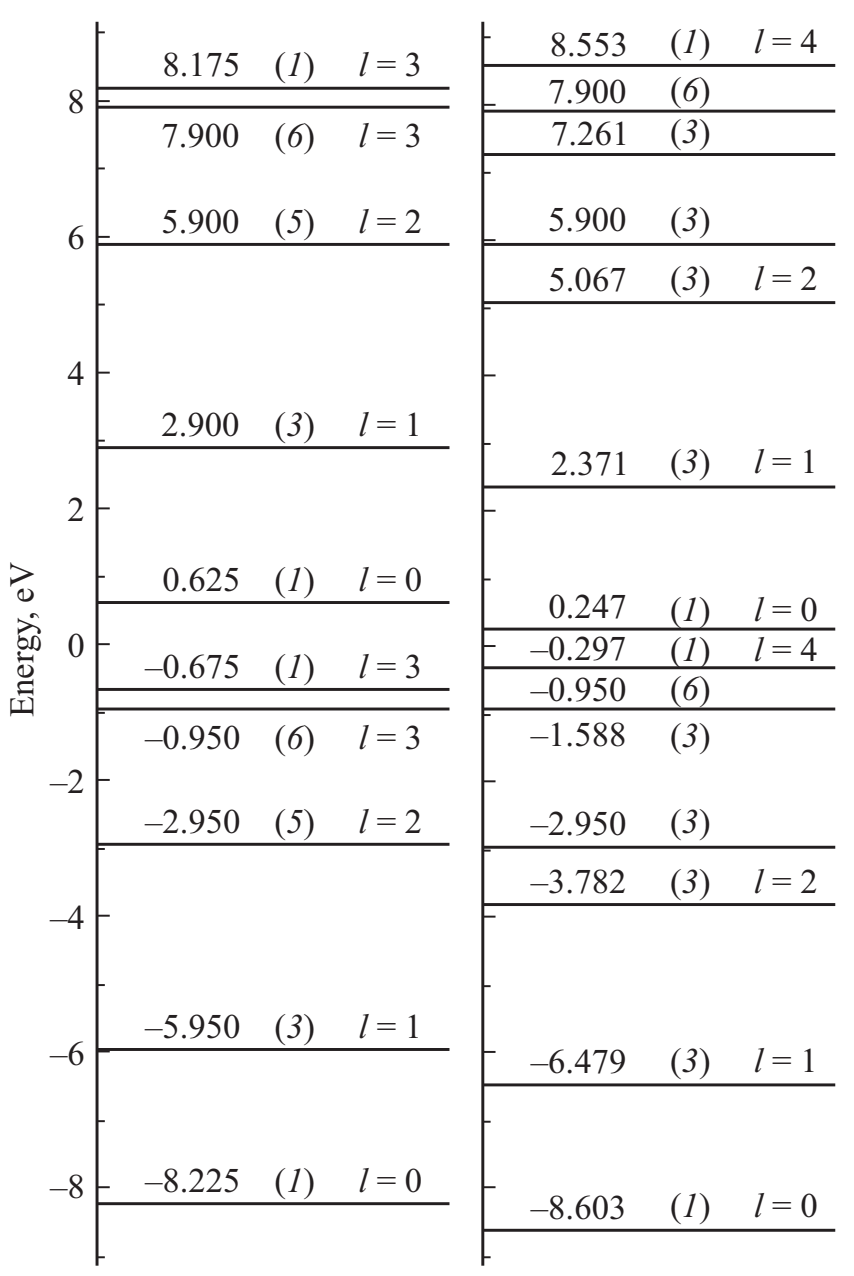

Pис. 8. Энергетический спектр фуллеренов $\mathrm{Au}_{16}$ (слева) и $\mathrm{Au}_{20}$ (справа) при значениях параметров системы: $U=8.85 \mathrm{eV}, B=-1 \mathrm{eV}, \varepsilon=-2.95 \mathrm{eV}$ с учетом кратностей вырождения.

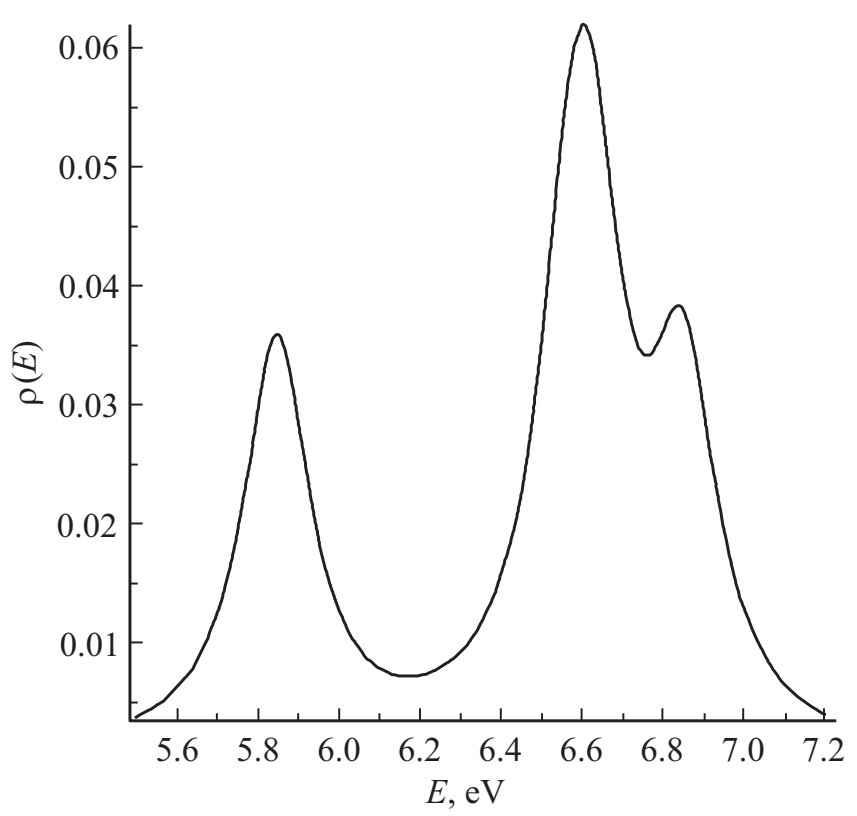

Рис. 9. Спектр оптического поглощения золотого фуллерена $\mathrm{Au}_{16}$ - зависимость интенсивности оптического поглощения от энергии фотонов в $\mathrm{eV}$.

зоны. Отметим, что в работе [18] золотой фуллерен $\mathrm{Au}_{20}$ назван магическим кластером, тогда как в магических кластерах электроны должны занимать все состояния, в случае $\mathrm{Au}_{20}$ не все $2 l+1=2 \cdot 4+1=9$ состояния заняты электронами. Тогда как в случае фуллерена $\mathrm{Au}_{16}$ все состояния в основном состоянии заняты электронами. При этом ширина зоны запрещенных энергий в случае $\mathrm{Au}_{16}$ больше по сравнению с $\mathrm{Au}_{20}$. Поэтому золотой фуллерен $\mathrm{Au}_{16} \mathrm{c}$ большим основанием может быть назван магическим кластером.

Оптические переходы являются разрешенными в основном состоянии и при низких температурах, если при поглощении или испускании кванта света изменение величины орбитального квантового числа $\Delta l= \pm 1$. Поскольку валентная зона полностью при $T=0$ и при низких температурах заполнена, оптическое поглощение возможно только при переходе электрона из нижней хаббардовской подзоны в верхнюю. С учетом этого спектр оптического поглощения имеет вид, приведенный на рис. 9. Все три пика поглощения соответствуют видимой области спектра.

Рассмотрим теперь спектр поглощения аниона $\mathrm{Au}_{16}^{-}$, представленный на рис. 10 слева. Два пика в спектре поглощения слева находятся в ближней инфракрасной области, третий пик справа принадлежит красному цвету видимой области света. Нас в первую очередь интересует инфракрасная область спектра поглощения. Дело в том, что излучение, соответствующее ближней инфракрасной области, пронизывает мягкие ткани и кровь живого организма, в частности человека, без поглощения ими излучения. Тогда инфракрасное излучение, соответствующее двум резонансным пикам 

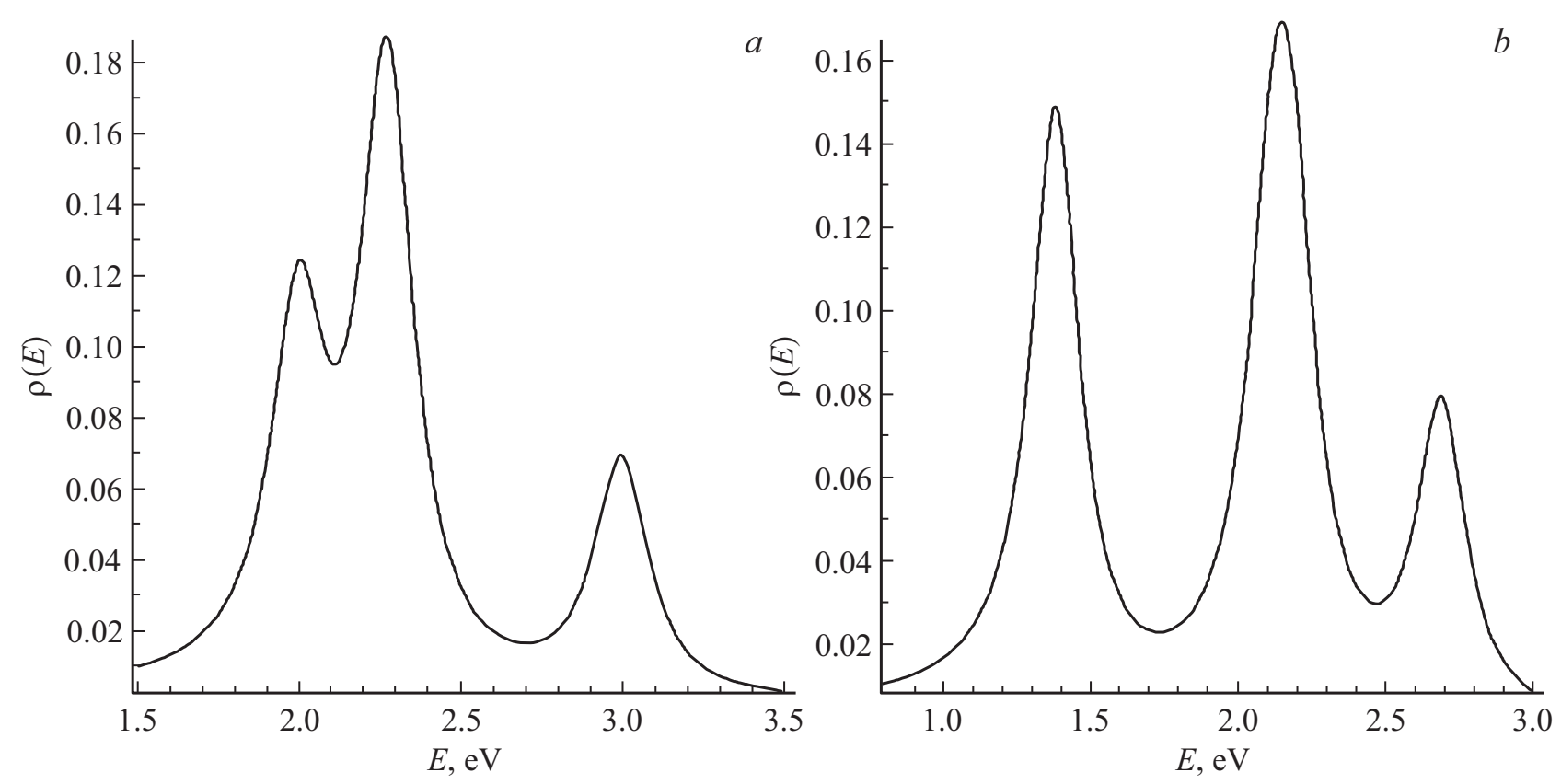

Рис. 10. Спектр оптического поглощения в ближней инфракрасной области спектра. Слева - спектр для иона фуллерена $А u_{16}^{-}$, справа - для иона фуллерена $\mathrm{Au}_{20}^{-}$.

слева легко пройдет через мягкие ткани и кровеносные сосуды и достигнет золотых фуллеренов, внедренных в организм. Если фуллерены из атомов золота окажутся сконцентрированными на поверхности раковых клеток, а этому может способствовать энергетическая выгодность перехода электрона с поверхности мембраны раковой клетки на молекулу фуллерена с образованием $\mathrm{Au}_{16}^{-}$, то рассматриваемые фуллерены можно будет использовать как новый класс материалов для получения контрастных улучшений при диагностике раковых заболеваний на ранних этапах. После подтверждения диагноза эти же ионы фуллерена из атомов золота можно использовать при лечении рака.

На рис. 10 справа приведен спектр поглощения ионами $\mathrm{Au}_{20}^{-}$. В этом случае все три резонансных пика находятся в ближней инфракрасной области, поэтому применение $\mathrm{Au}_{20}^{-}$при диагностике и терапии раковых заболеваний даже предпочтительнее по сравнению с $\mathrm{Au}_{16}^{-}$.

Таким образом, в рамках квантовой теории поля удается описать основные физико-химические свойства фуллеренов $\mathrm{Au}_{16}$ и $\mathrm{Au}_{20}$. Спектр оптического поглощения электроотрицательных ионов фуллерена указывает на возможность их применения при диагностике и лечении раковых заболеваний.

\section{Список литературы}

[1] L. Au, J. Chen, L.V. Wang, Y. Xia. Canser Nanotechnology. Meth. Mol. Biol. 624, 83 (2010).

[2] S. Bulusu, X. Li, L. Wang, X.G. Zeng. Proc. Natl. Acad. Sci. U.S.A. 103, 8326 (2006).

[3] G. Schmid, U. Simon. Chem. Commun. 6, 697 (2005).
[4] M. Homberger, U. Simon. Phil. Trans. R. Soc. A 368, 1405 (2010).

[5] Y. Okinaka. Gold Bull. 33, 117 (2000).

[6] P. Gruene, D.M. Rayner, B. Redlich, A.F.G. van der Meer, J.T. Lyon, G. Meijer, A. Fielicke. Science 321, 674 (2008).

[7] M.E. Stewart, C.R. Anderton, L.B. Thompson, J. Maria, S.K. Gray, J.A. Rogers, R.G. Nuzzo. Chem. Rev. 108, 494 (2008).

[8] D.I. Gittins, D. Bethell, D.J. Schiffrin, R.J. Nichols. Nature 408 , 67 (2000).

[9] M. Haruta, T. Kobayashi, H. Sano, N. Yamada. Chem. Lett. 16, 405 (1987).

[10] M. Haruta, N. Yamada, T. Kobayashi, S. Iijima. J. Catal. 115, 301 (1989).

[11] M.A. Mackey, M.A. Matmoues, M.A. El-Sayed. Bioconjugate Chem. 24, 897 (2013).

[12] S. Her, D.A. Jaaffray, C. Allen. Adv. Drug Delver. 109, 84 (2017).

[13] W. Li, P.K. Brown, L.V. Wang, Y. Xi. Contrast Media Mol. Imaging. 6, 370 (2011).

[14] A. Zhang, W. Guo, J. Wang, X. Ma, D. Yu. Nanoscale Res. Lett. 11, 279 (2016).

[15] S.E. Skrabalak, J. Chen, Y. Sun, X. Lu, L. Au, C.M. Cobley, Y. Xia. Accounts Chem. Res. 41, 1587 (2008).

[16] Г.И. Миронов. ФТТ 50, 182 (2008).

[17] Г.И. Миронов. ФММ 105, 355 (2008).

[18] E.S. Kryachko, F. Remacle. Int. J. Quantum Chem. 107, 2922 (2007).

[19] K.J. Taylor, C.L. Pettiette-Hall, R.E. Smalley. J. Chem. Phys. 96, 3319 (1992).

[20] C. Jackschath, I. Rabin, W. Schulz. Phys. Chem. 86, 1200 (1992).

[21] J. Li, X. Li, H.-J. Zhai, L.-S. Wang. Science 299, 864 (2003). 
[22] П.Н. Дьячков. Журн. неорган. химии 60, 1045 (2015).

[23] Г.И. Миронов. Журн. неорган. химии 63, 72 (2018).

[24] J. Hubbard. Proc. Roy. Soc. A 276, 238 (1963).

[25] S.P. Shubin, S.V. Wonsowskii. Proc. Roy. Soc. A 145, 159 (1934).

[26] С.В. Тябликов. Методы квантовой теории магнетизма. Наука, М. (1965). 335 c.

[27] E.M. Fernandez, J.M. Soler, I.L. Garzon, L.C. Balbas. Phys. Rev. B 70, 165403 (2004).

[28] J. Wang, C. Wang, J. Zhao. Chem. Phys. Lett. 380, 716 (2003).

[29] J. Li, X. Li, H.J. Zhai, L.-S. Wang. Science 299. 864 (2003).

Редактор Т.Н. Василевская 